\title{
Comparison of HE4, CA125, ROMA and CPH-I for Preoperative Assessment of Adnexal Tumors
}

\author{
Núria Carreras-Dieguez ${ }^{1}$, Ariel Glickman ${ }^{1}$, Meritxell Munmany ${ }^{1,2}$, Georgina Casanovas ${ }^{3}{ }^{[0}$, Núria Agustí ${ }^{1}$, \\ Berta Díaz-Feijoo 1,2,4, Adela Saco ${ }^{5,6}$, Beatriz Sánchez ${ }^{1}$, Lydia Gaba ${ }^{7,8}$, Martina Aida Angeles 9 (®),

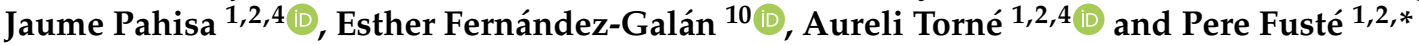

check for updates

Citation: Carreras-Dieguez, N.; Glickman, A.; Munmany, M.;

Casanovas, G.; Agustí, N.;

Díaz-Feijoo, B.; Saco, A.; Sánchez, B.; Gaba, L.; Angeles, M.A.; et al. Comparison of HE4, CA125, ROMA and $\mathrm{CPH}-\mathrm{I}$ for Preoperative Assessment of Adnexal Tumors. Diagnostics 2022, 12, 226. https:// doi.org/10.3390/diagnostics12010226 Academic Editor: Edward J. Pavlik

Received: 9 December 2021 Accepted: 14 January 2022 Published: 17 January 2022

Publisher's Note: MDPI stays neutral with regard to jurisdictional claims in published maps and institutional affiliations.

Copyright: (c) 2022 by the authors. Licensee MDPI, Basel, Switzerland. This article is an open access article distributed under the terms and conditions of the Creative Commons Attribution (CC BY) license (https:// creativecommons.org/licenses/by/ $4.0 /)$.
1 Gynecologic Oncology Unit, Clinic Institute of Gynecology, Obstetrics, and Neonatology, Hospital Clínic of Barcelona, 08036 Barcelona, Spain; ncarreras@clinic.cat (N.C.-D.); glickman@clinic.cat (A.G.); mmunmany@clinic.cat (M.M.); nagusti@clinic.cat (N.A.); bdiazfe@clinic.cat (B.D.-F.); besanchez@clinic.cat (B.S.); jpahisa@clinic.cat (J.P.); atorne@clinic.cat (A.T.)

2 Faculty of Medicine, University of Barcelona, 08036 Barcelona, Spain

3 Medical Statistics Core Facility, Institut d'Investigacions Biomèdiques August Pi i Sunyer (IDIBAPS), 08036 Barcelona, Spain; gcasanovas@clinic.cat

4 Gynecology Oncology Unit, Institute Clinic of Gynecology, Obstetrics and Neonatology, Hospital Clínic de Barcelona, Institut d'Investigacions Biomèdiques August Pi i Sunyer (IDIBAPS), Faculty of Medicine, University of Barcelona, 08036 Barcelona, Spain

5 Institut de Salut Global de Barcelona (ISGlobal), 08036 Barcelona, Spain; masaco@clinic.cat

6 Gynecology Oncology Unit, Department of Pathology, Hospital Clínic of Barcelona, 08036 Barcelona, Spain

7 Gynecology Oncology Unit, Department of Medical Oncology, Hospital Clínic of Barcelona, 08036 Barcelona, Spain; lgaba@clinic.cat

8 Translational Genomics and Targeted Therapeutics in Solid Tumors, Institut d'Investigacions Biomèdiques August Pi i Sunyer (IDIBAPS), 08036 Barcelona, Spain

9 Department of Surgical Oncology, Institut Claudius Regaud, Institut Universitaire du Cancer de Toulouse (IUCT), Oncopole, 31100 Toulouse, France; angeles@clinic.cat

10 Biomedical Diagnostic Center, Hospital Clínic of Barcelona, 08036 Barcelona, Spain; esfernandez@clinic.cat

* Correspondence: pfuse@clinic.cat

Abstract: (1) OBJECTIVE: To assess the performance of CA125, HE4, ROMA index and CPH-I index to preoperatively identify epithelial ovarian cancer (EOC) or metastatic cancer in the ovary (MCO). (2) METHODS: single center retrospective study, including women with a diagnosis of adnexal mass. We obtained the AUC, sensitivity, specificity and predictive values were of HE4, CA125, ROMA and $\mathrm{CPH}-\mathrm{I}$ for the diagnosis of EOC and MCO. Subgroup analysis for women harboring adnexal masses with inconclusive diagnosis of malignancy by ultrasound features and Stage I EOC was performed. (3) RESULTS: 1071 patients were included, 852 (79.6\%) presented benign/borderline tumors and 219 (20.4\%) presented EOC/MCO. AUC for HE4 was higher than for CA125 (0.91 vs. 0.87). No differences were seen between AUC of ROMA and CPH-I, but they were both higher than HE4 AUC. None of the tumor markers alone achieved a sensitivity of $90 \%$; HE4 was highly specific (93.5\%). ROMA showed a sensitivity and specificity of $91.1 \%$ and $84.6 \%$ respectively, while CPH-I showed a sensitivity of $91.1 \%$ with $79.2 \%$ specificity. For patients with inconclusive diagnosis of malignancy by ultrasound features and with Stage I EOC, ROMA showed the best diagnostic performance (4) CONCLUSIONS: ROMA and CPH-I perform better than tumor markers alone to identify patients harboring EOC or MCO. They can be helpful to assess the risk of malignancy of adnexal masses, especially in cases where ultrasonographic diagnosis is challenging (stage I EOC, inconclusive diagnosis of malignancy by ultrasound features).

Keywords: tumor markers; HE4; Ca125; ROMA; CPH-I; adnexal tumors

\section{Introduction}

Epithelial ovarian cancer (EOC) is the leading cause of death in patients with gynecological malignancies [1]. Most cases are diagnosed in advanced stages, since screening 
has not shown to be beneficial, and the disease has an insidious onset with unspecific presenting symptoms. Pelvic adnexal masses are common in female population, yet only a small percentage represent ovarian malignancies [2]. While benign ovarian masses can be managed in non-specialized centers, patients with EOC or metastatic cancer in the ovary (MCO) should be treated by a multidisciplinary experienced team [3]. Surgical staging for suspected early-stage EOC is a complex procedure. Moreover, it has been demonstrated that, in advanced EOC, there is an increase in overall survival when cytoreductive surgery is performed by a specialized team in gynecologic oncology [3]. Therefore, an accurate differential diagnosis and referral to specialized centers of women harboring suspicious adnexal masses is critical to enhancing their survival.

Preoperatively, ultrasound and serum tumor markers can help to identify patients with adnexal masses that harbor a high risk of EOC or MCO. Expert ultrasonography examination plays an important role in the detection of EOC [4,5], but it is challenging in early stages and is not available in all centers. IOTA simple rules are 10 validated ultrasound-based rules allowing to classify adnexal masses in benign, malignant, or unclassifiable; several studies have reported a prevalence of unclassifiable adnexal masses of $22-33 \%$ [6-8]. The serum tumor marker cancer antigen 125 (CA125) has been traditionally used as a tool for diagnosis and follow-up for EOC patients [9]. However, CA125 serum levels are increased in less than half of early-stage EOC cases, and also raise in many other benign or malignant medical conditions [10], resulting in a decreased sensitivity and specificity [11]. Human epididymis protein 4 (HE4) is a serum tumor marker introduced during the last decade for EOC diagnosis. It has shown to improve sensitivity and specificity for detection of EOC over CA125, especially in premenopausal women and in early stages of EOC [11]. Unlike CA125, HE4 is not overexpressed in benign ovarian disease or normal ovarian tissue [12]. HE4 levels increase progressively with advancing age, which raises an issue when defining its reference range [13]. Serum HE4 levels have shown to be increased in other pathological conditions such as lung cancer, endometrial cancer, or renal failure [14].

Several models have been developed to determine the risk of malignancy of adnexal masses. Such tools are based on different combinations of clinical data, ultrasound features, and serum tumor markers. The Risk of Ovarian Malignancy Algorithm (ROMA), described in 2009 by Moore et al. [15], combines serum levels of HE4 and CA125 with menopausal status to obtain a probability risk of harboring EOC. A recent meta-analysis has shown a sensitivity of $87 \%$ and a specificity of $86 \%$ [16]. In 2015, Karlsen et al., developed the Copenhagen Index (CPH-I), based as well on serum HE4 and CA125, but combined with age [17]. CPH-I model has shown a sensitivity of $69 \%$ and specificity of $85 \%$ to differentiate benign from malignant and borderline tumors [18].

Several studies evaluating the role of HE4, CA125, and ROMA in preoperative assessment of adnexal masses have been published [14,16,19-21]. However, scarce literature regarding the performance of CPH-I can be found [18,22,23]. Furthermore, there is little information about the role of ROMA and CPH-I in the evaluation of specific subgroups of patients in which the differential diagnosis of adnexal tumors is particularly challenging, such as women harboring adnexal masses with an inconclusive diagnosis by ultrasonographic examination, women with Stage I EOC (whose diagnosis is difficult by ultrasound [24], or premenopausal women, in which tumor markers have shown worse performance compared to postmenopausal women [19]). Thus, it remains unclear which is the best approach for preoperative evaluation of adnexal masses.

The main objective of this study was to assess the performance of CA125, HE4, ROMA, and CPH-I on the specific detection of adnexal masses who will benefit from derivation to a reference center, prioritization in surgery waiting list and surgery performance by surgeons specialized in gynecologic oncology, that is EOC and MCO. Our secondary objectives were to evaluate the role of CA125, HE4, ROMA, and CPH-I to diagnose EOC or MCO in three challenging situations: premenopausal women, Stage I EOC and adnexal masses with an inconclusive diagnosis of malignancy by ultrasound features, using IOTA simple rules. 


\section{Materials and Methods}

We performed an observational retrospective study, including women consecutively referred to the Gynecological Oncology Unit of Hospital Clínic de Barcelona with a diagnosis of ovarian cyst or pelvic mass between January 2000 and December 2018. Inclusion criteria were: (1) patients with an ovarian cyst or pelvic mass identified in pelvic imaging (ultrasound, CT scan, RMI); (2) tumor markers and ultrasound performed preoperatively; (3) pathology results available and evaluated in our center, confirming benign ovarian disease, borderline epithelial ovarian tumors, EOC or MCO. Patients without pathology results available or harboring other synchronic malignancies were excluded. Patients harboring non-epithelial ovarian cancer or non-ovarian masses were excluded from the analysis.

We defined two study groups according to pathology findings. Group A comprised women harboring benign pathology and borderline epithelial ovarian tumors. Group $\mathrm{B}$ included women diagnosed with EOC or MCO. This classification was based on the differences in the management of adnexal masses between these groups: patients with EOC or MCO benefit from derivation to a reference center, prioritization in surgery waiting list and surgery performance by surgeons specialized in gynecologic oncology.

Data were collected from clinical records. The preoperative results of blood tests were retrieved from medical records. Serum levels of CA125 and HE4 were measured using a chemiluminescent enzyme immunoassay on the ADVIA Centaur ${ }^{\circledR}$ XP (Siemens Healthcare Diagnostics Inc., Tarrytown, NY, USA) and the Architect ${ }^{\circledR}$ Analyzer (Abbott Laboratories, Chicago, IL, USA), respectively; as part of our institution's usual clinical practice. All samples were stored, and the tests were performed according to the manufacturer's instructions; the controls were within the ranges provided. HE4 was not considered in patients with impaired kidney function (serum creatinine levels higher than $1.3 \mathrm{mg} / \mathrm{dL}$ or glomerular filtration rate minor to $90 \mathrm{~mL} / \mathrm{min}$ ). Index values (ROMA and CPH-I) were computed for all patients according to the published algorithms [15,17]. Menopause was defined as cessation of menstruation for at least 12 months. Transvaginal ultrasound by an expert ultrasonographer is always performed preoperatively in patients harboring an adnexal mass as part of our institution protocol. Adnexal masses were classified as probably benign, probably malignant, or inconclusive diagnosis according to IOTA simple rules [6]. All the included patients underwent surgery after diagnosis of the adnexal mass. Patients with suspected malignancy underwent surgery less than 1 month after diagnosis, whereas patients without suspected malignancy underwent surgery less than 3 years after diagnosis. Pathology analysis of removed or biopsied adnexal masses was performed according to 2014 WHO classification of tumors of the female reproductive organs [25]. The value of tumor markers was available to pathologists at the time of evaluation of adnexal masses.

Categorical variables were reported as absolute frequencies $(n)$ and proportions $(\%)$, and continuous variables as mean \pm standard deviation or median [interquartile range]. The receiver operating characteristic (ROC) curves for tumor markers, $\mathrm{CPH}-\mathrm{I}$ and ROMA were plotted, and the diagnostic accuracy, sensitivity, specificity, positive predictive value $(\mathrm{PPV})$ and negative predictive value (NPV) and their $95 \%$ confidence intervals $(\mathrm{CI})$ were calculated to evaluate the diagnostic performance of CA125, HE4, ROMA, and CPH-I at different cut-off points reported by literature [15-18,22]. AUCs were compared using the Delong nonparametric approach [26]. We performed a subgroup analysis including only premenopausal, postmenopausal women, Stage I EOC, and women harboring adnexal masses with an inconclusive diagnosis of malignancy by ultrasound features using IOTA simple rules [27].

Statistical analysis was performed using STATA/IC version 15.1 (College Station, TX USA) and SAS version 9.4 (SAS Institute Inc., Cary, NC, USA). The level of significance was set at two-sided $5 \%$ (i.e., 0.05 ).

\section{Results}

During the study period, 1124 patients were referred to our center because of adnexal masses. We excluded 31 patients because serum tumor markers had not been obtained 
preoperatively, three patients because they presented other synchronic malignancies, and four patients because of surgical findings of non-adnexal pathology. Twelve patients had impaired kidney function. Sixteen patients had non-epithelial ovarian cancer and were excluded as well. Finally, 1071 patients were included. Table 1 summarizes the main characteristics of the studied population.

Table 1. General characteristics of the study population.

\begin{tabular}{cccc}
\hline & $N=1071$ & & \\
\hline Age (mean \pm SD) & $47.72 \pm 16.22$ & & \\
Menopausal status & & & \\
Premenopausal & $629(58.73 \%)$ & \\
Postmenopausal & $442(41.27 \%)$ & & \\
Pathology & $778(72.64 \%)$ & & \\
Benign & $331(42.5 \%)$ & & \\
Epithelial tumor & $210(26.9 \%)$ & & \\
Endometrioma & $56(7.2 \%)$ & & \\
Fibroids & $4(0.5 \%)$ & & \\
Other sex cord-stromal tumors & $149(19.2 \%)$ & & \\
Germ cell tumors & $24(3.1 \%)$ & & \\
Fallopian tube lesions & $4(0.5 \%)$ & FIGO stage for EOC \\
Tubo-ovaric abscesses & $74(6.91 \%)$ & & \\
Epithelial borderline & $197(18.39 \%)$ & & \\
Epithelial ovarian cancer (EOC) & $124(62.94 \%)$ & & \\
Serous carcinoma & $8(4.06 \%)$ & & \\
Mucinous carcinoma & $17(8.63 \%)$ & FIGO Stage III & \\
Clear cell carcinoma & $33(16.75 \%)$ & FIGO Stage IV & \\
Endometrioid Carcinoma & $15(7.61 \%)$ & & \\
Undifferenciated Carcinoma & $22(2.05 \%)$ & & \\
Metastatic cancer in the ovary & & &
\end{tabular}

Eight-hundred fifty-two (79.6\%) patients presented benign or borderline epithelial tumors (Group A) and 219 (20.4\%) presented EOC or MCO (Group B). One hundred and ninety-seven patients harbored EOC, fifty-eight of whom had FIGO Stage I. Serous carcinoma was the most frequent type of EOC, followed by endometrioid carcinoma. Twenty-two patients $(2.05 \%)$ harbored MCO. Seventy-four patients had epithelial borderline tumors. Premenopausal women accounted for $58.7 \%$ of the sample, while there were $41.3 \%$ postmenopausal women. In 348 patients ( $32.58 \%$ ), there was an inconclusive diagnosis by ultrasound features using IOTA simple rules. The mean age of patients in Group A was significantly lower that in Group B $(44.8 \pm 0.53$ vs. $59.6 \pm 0.88$ years, $p<0.05)$. The proportion of premenopausal patients was higher in Group A (67.3\% vs. $25.1 \%)$. There were no differences in kidney function (creatinine levels) between study groups $(p<0.19)$.

For Group B patients' median values of CA125 and HE4 were 184 (49-671) U/mL and 246 (104.7-765.5) pmol/L, respectively. For Group A patients, they were 13 (8- 30) U/mL and $45.5(35.4-57.1) \mathrm{pmol} / \mathrm{L}$. The difference between groups was statistically significant for both tumor markers. The predicted probability of malignancy of ROMA (0.85 vs. 0.07) and $\mathrm{CPH}-\mathrm{I}$ (0.68 vs. 0.01 ) was also higher in Group B. No adverse events derived from tumor marker testing were registered.

Table 2 and Figure 1 show the results of ROC analysis for detecting EOC or MCO. The area under the curve (AUC) for HE4 was significantly higher than AUC for CA125 $(p<0.05)$. Non-significant differences were seen between the AUC of ROMA and CPH-I, but they were both higher than HE4 AUC $(p<0.05)$. Subgroup analysis showed that in premenopausal women, HE4 performed better than CA125 $(p<0.05)$, and was equivalent to ROMA or CPH-I. In postmenopausal women, HE4 and CA125 AUCs showed no differences, but both ROMA and CPH-I performed better than the tumor markers alone $(p<0.05)$. Considering only adnexal masses with an inconclusive diagnosis using IOTA simple rules, ROMA performed significantly better than CPH-I and tumor markers alone $(p<0.05)$. 
With regard to the diagnosis of Stage I EOC, ROMA and CPH-I performed similarly, and ROMA performed significantly better than CA125 and HE4 alone $(p<0.05)$. We found no significant differences in the AUC of HE4 and CPH-I in Stage I EOC. Supplemental Table S1 summarizes the main findings of ROC analysis.

Table 2. ROC analysis of Ca125, He4, ROMA and CPH for diagnosis of EOC or MCO. Area under the curve (95\% CI).

\begin{tabular}{cccccc}
\hline & All Patients & $\begin{array}{c}\text { Inconclusive } \\
\text { Diagnosis * }\end{array}$ & Premenopausal & Postmenopausal & Stage I EOC \\
\hline \multirow{2}{*}{ CA125 } & 0.873 & 0.810 & 0.759 & 0.933 & 0.810 \\
HE4 & $(0.842-0.904)$ & $(0.743-0.877)$ & $(0.675-0.842)$ & $(0.907-0.959)$ & $(0.751-0.869)$ \\
& 0.909 & 0.844 & 0.863 & 0.905 & 0.856 \\
ROMA & $0.881-0.938)$ & $(0.779-0.910)$ & $(0.791-0.934)$ & $(0.870-0.940)$ & $(0.793-0.8869)$ \\
& $(0.916-0.962)$ & $(0.846-0.941)$ & $(0.796-0.937)$ & $(0.937-0.975)$ & $(0.863-0.955)$ \\
CPH & 0.936 & 0.876 & 0.860 & 0.955 & 0.901 \\
& $(0.913-0.958)$ & $(0.822-0.931)$ & $(0.793-0.928)$ & $(0.935-0.975)$ & $(0.855-0.947)$ \\
\hline
\end{tabular}

* Inconclusive diagnosis of malignancy by ultrasound features using IOTA simple rules.

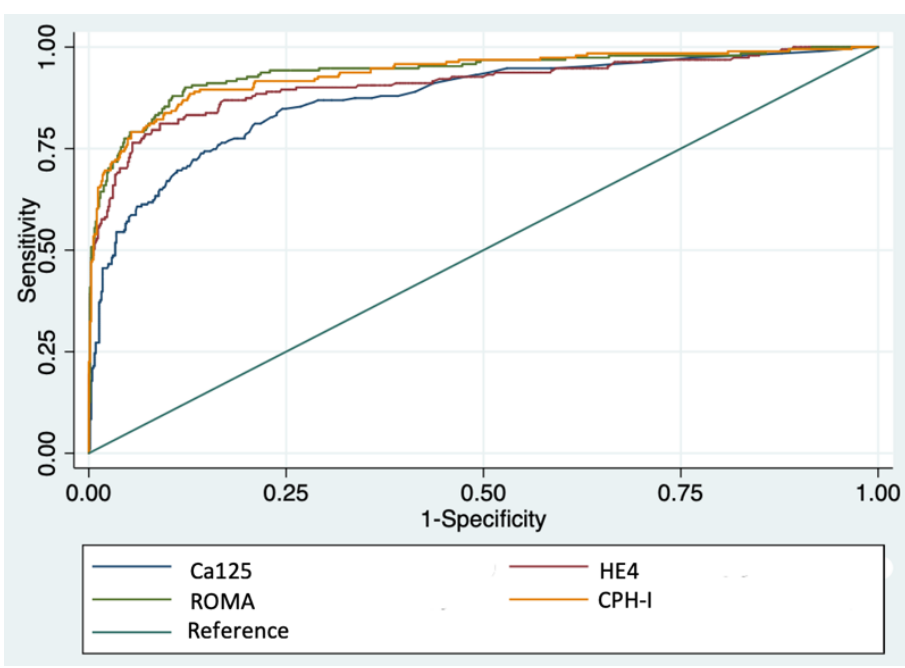

Figure 1. ROC curve of Ca125, He4, ROMA and CPH for diagnosis of EOC or MCO.

Table 3 shows sensitivity, specificity, and PPV and NPV of CA125, HE4, ROMA, and $\mathrm{CPH}-\mathrm{I}$ at different cutoff points previously reported by literature. None of the tumor markers alone achieved a sensitivity of $90 \%$; HE4 at a cutoff point of 70 for premenopausal women and 140 for postmenopausal women, was highly specific (93.5\%). ROMA index at a cutoff point of 15 showed a sensitivity and specificity of $91.1 \%$ and $84.6 \%$ respectively and of $91.6 \%$ and $82.6 \%$ at a cutoff point of $12.5 / 14.4$ for pre/postmenopausal women; in both cases, PPV was higher than 50\%. CPH-I at a cutoff point of three showed a sensitivity of $91.1 \%$ with $79.2 \%$ specificity and PPV of $54.72 \%$.

False negative results for CA125, HE4, ROMA and CPH-I were found in 53, 38, 8 and 20 patients, respectively. Supplemental Table S2 summarizes histological tumor types of patients with false negative results. For CA125 and HE4, most patients with false negative results harbored serous carcinoma. The most common false negative result for ROMA and CPH-I was obtained in patients with metastatic cancer in the ovary. More than $70 \%$ of patients with false negative results of tumor markers or probabilistic indexes suffered from early-stage EOC. Regarding false positive results, $62.7 \%(n=96)$ of patients with false positive result for CA125 determination harbored endometriomas. This proportion was lower for ROMA (14.1\%), CPH-I (19.35\%) and HE4 (3.7\%, $n=1)$. Nineteen $(9.9 \%)$ patients in Group B had negative HE4 (<120 pmol/L) but positive CA125(>100 U/mL), while 30 (15.7\%) patients had negative CA125 but positive HE4. 
Table 3. Sensitivity, specificity, and predictive values of Ca125, He4, ROMA index and CPH-1 index at different cutoff points.

\begin{tabular}{|c|c|c|c|c|c|}
\hline Parameter & Cutoff & $\begin{array}{c}\text { Sensitivity } \\
(95 \% \text { CI })\end{array}$ & $\begin{array}{c}\text { Specificity } \\
\text { (95\% CI) }\end{array}$ & PPV (95\% CI) & NPV $(95 \%$ CI $)$ \\
\hline \multirow{2}{*}{ CA125 } & $100 \mathrm{U} / \mathrm{mL}$ & $\begin{array}{c}61.86 \\
(55.21-68.09)\end{array}$ & $\begin{array}{c}92.71 \\
(90.73-94.29)\end{array}$ & $\begin{array}{c}68.91 \\
(62.07-75.02)\end{array}$ & $\begin{array}{c}90.30 \\
(88.11-92.11)\end{array}$ \\
\hline & $35 / 65 \mathrm{U} / \mathrm{mL}^{*}$ & $\begin{array}{c}74.42 \\
(68.23-79.81)\end{array}$ & $\begin{array}{c}80.70 \\
(77.81-83.24)\end{array}$ & $\begin{array}{c}50.21 \\
(44.73-55.64)\end{array}$ & $\begin{array}{c}92.30 \\
(90.22-94.12)\end{array}$ \\
\hline \multirow{4}{*}{$\mathrm{HE} 4$} & $35 / 100 \mathrm{U} / \mathrm{mL}^{*}$ & $\begin{array}{c}66.98 \\
(60.44-72.92)\end{array}$ & $\begin{array}{c}81.39 \\
(78.58-83.90)\end{array}$ & $\begin{array}{c}48.48 \\
(42.86-54.15)\end{array}$ & $\begin{array}{c}90.41 \\
(88.07-92.32)\end{array}$ \\
\hline & $70 \mathrm{pmol} / \mathrm{L}$ & $\begin{array}{c}83.25 \\
(77.31-87.88)\end{array}$ & $\begin{array}{c}86.11 \\
(83.40-88.43)\end{array}$ & $\begin{array}{c}61.15 \\
(55.11-66.87)\end{array}$ & $\begin{array}{c}95.14 \\
(93.22-96.53)\end{array}$ \\
\hline & $120 \mathrm{pmol} / \mathrm{L}$ & $\begin{array}{c}69.11 \\
(62.23-75.23)\end{array}$ & $\begin{array}{c}96.29 \\
(94.65-97.44)\end{array}$ & $\begin{array}{c}83.02 \\
(76.42-88.06)\end{array}$ & $\begin{array}{c}92.23 \\
(90.10-93.93)\end{array}$ \\
\hline & $70 / 140 \mathrm{pmol} / \mathrm{L} *$ & $\begin{array}{c}70.70 \\
(63.93-76.71)\end{array}$ & $\begin{array}{c}93.5 \\
(91.52-95.11)\end{array}$ & $\begin{array}{c}74.2 \\
(67.40-80.01)\end{array}$ & $\begin{array}{c}92.40 \\
(90.20-94.10)\end{array}$ \\
\hline \multirow[b]{2}{*}{ ROMA } & $10 \%$ & $\begin{array}{c}94.24 \\
(89.98-96.75)\end{array}$ & $\begin{array}{c}72.71 \\
(69.27-75.91)\end{array}$ & $\begin{array}{c}47.91 \\
(43.84-54.01)\end{array}$ & $\begin{array}{c}97.85 \\
(96.19-98.80)\end{array}$ \\
\hline & $15 \%$ & $\begin{array}{c}91.10 \\
(86.21-94.37)\end{array}$ & $\begin{array}{c}84.62 \\
(81.73-87.12)\end{array}$ & $\begin{array}{c}62.14 \\
(56.33-67.62)\end{array}$ & $\begin{array}{c}97.17 \\
(95.51-98.22)\end{array}$ \\
\hline \multirow{6}{*}{$\mathrm{CPH}$} & $12.5 / 14.4 \%$ * & $\begin{array}{c}91.62 \\
(86.83-94.78)\end{array}$ & $\begin{array}{c}82.58 \\
(79.57-85.23)\end{array}$ & $\begin{array}{c}59.32 \\
(53.63-64.77)\end{array}$ & $\begin{array}{c}97.26 \\
(95.60-98.31)\end{array}$ \\
\hline & $13.1 / 27.7 \%$ * & $\begin{array}{c}84.80 \\
(79.00-89.20)\end{array}$ & $\begin{array}{c}89.0 \\
(86.40-91.10)\end{array}$ & $\begin{array}{c}68.10 \\
(61.90-73.70)\end{array}$ & $\begin{array}{c}95.50 \\
(93.60-96.80)\end{array}$ \\
\hline & $1 \%$ & $\begin{array}{c}96.86 \\
(93.32-98.55)\end{array}$ & $\begin{array}{c}47.97 \\
(44.26-51.70)\end{array}$ & $\begin{array}{c}34.01 \\
(30.15-38.09)\end{array}$ & $\begin{array}{c}98.22 \\
(96.17-99.18)\end{array}$ \\
\hline & $3 \%$ & $\begin{array}{c}91.10 \\
(86.22-94.42)\end{array}$ & $\begin{array}{c}79.12 \\
(75.91-82.01)\end{array}$ & $\begin{array}{c}54.72 \\
(49.23-60.14)\end{array}$ & $\begin{array}{c}97.01 \\
(95.21-98.12)\end{array}$ \\
\hline & $5 \%$ & $\begin{array}{c}86.91 \\
(81.39-90.97)\end{array}$ & $\begin{array}{c}87.54 \\
(84.86-89.79)\end{array}$ & $\begin{array}{c}65.87 \\
(59.82-71.45)\end{array}$ & $\begin{array}{c}96.03 \\
(94.20-97.29)\end{array}$ \\
\hline & $7 \%$ & $\begin{array}{c}82.20 \\
(76.16-86.97)\end{array}$ & $\begin{array}{c}90.87 \\
(88.49-92.80)\end{array}$ & $\begin{array}{c}71.36 \\
(65.06-76.93)\end{array}$ & $\begin{array}{c}94.86 \\
(92.90-96.309)\end{array}$ \\
\hline
\end{tabular}

PPV: positive predictive value; NPV: negative predictive value * premenopausal/postmenopausal women.

\section{Discussion}

In the present study, ROMA and CPH-I performed similarly to identify EOC or MCO. The algorithms performed significantly better than tumor markers alone. ROMA index includes menopausal status, whose definition is not the same in all studies and may sometimes be unknown [15]. CPH-I is independent of menopausal status and includes age, which is easier to obtain [17]. The diagnostic performance of isolated tumor markers and probabilistic indexes was different in the subgroups of premenopausal patients, patients with inconclusive ultrasonographic diagnosis and for the detection of Stage I EOC.

\subsection{Diagnostic Performance of Tumor Markers and Probabilistic Indexes in Overall Population}

The authors of the ESGO/ISUOG/IOTA/ESGE Consensus Statement on Preoperative Diagnosis of Ovarian Tumors state that neither HE4 nor ROMA improve the discrimination between benign and malignant masses compared to HE4 alone [28]. Comparing the sensitivity and specificity of the tumor markers and the probability indexes with those reported in the literature is challenging $[16,18,21]$, as the cutoff points used by different groups are not always the same, and neither are the comparison groups according to pathological findings (e.g., in some publications, borderline tumors or non-epithelial ovarian cancer are included in the malignancy group and compared to benign ovarian tumors $[18,21])$. The sensitivity and specificity values of ROMA and CPH-I shown in Table 3 are consistent with those previously reported. Moore et al. [29] reported sensitivity for ROMA of $94 \%$ at a set specificity of $75 \%$ at a cutoff point $12.9 / 27.8$ (pre/postmenopausal), while in a meta-analysis by Dayyani et al. [16] a sensitivity of $87.3 \%$ and a specificity of $85.5 \%$ are reported (different cutoff values depending on the study). In the validation cohort of Karlsen et al., a sensitivity of $82.0 \%$ and specificity of $88.4 \%$ for CPH-I at a cutoff of 7 were found [17].

There are few studies in the literature comparing ROMA and CPH-I. The study of Minar et al. [18] reported a sensitivity and specificity of $71 \%$ and $88 \%$ for ROMA (cutoff 
point 13.1/27.7 for pre/postmenopausal) and of $69 \%$ and $85 \%$ for CPH-I (cutoff point 7). The study of Yoshida et al. [22] described sensitivity of $71.2 \%$ and specificity of $83.5 \%$ for ROMA (cutoff $13.1 / 27.7$ ), and of $73.1 \%$ and $84.4 \%$ for CPH-I (cutoff point 7 ). In our study, sensitivity and specificity at these cutoff points are slightly higher. Similarly, Tu Tran et al. recently reported a sensitivity and specificity of $74.2 \%$ and $91.8 \%$ for ROMA (cutoff 16.5 ) and $87.1 \%$ and $78.5 \%$ for CPH-I (cutoff 1.89 ), respectively. The authors of the latter work did not find significant differences between the diagnostic performance of ROMA and CPH-I [23].

\subsection{Diagnostic Performance of Tumor Markers in Specific Subgroups of Patients}

In premenopausal women, HE4 performed significantly better than CA125 and was equivalent to ROMA or CPH-I, highlighting the value of this tumor marker in this subgroup of patients. This finding is supported by previous studies that suggest that in premenopausal women ROMA does not seem to have any advantage over the use of HE4 [30-32]. A recent meta-analysis retrieving data from 32 studies showed higher AUC and specificity of HE4 in premenopausal women, compared to CA125 and ROMA [33]. Other authors report higher AUCs for CPH-I, when compared to ROMA and HE4 in premenopausal women [23].

CA125 can raise in patients with endometriosis, thus differentiating ovarian endometriosis from EOC in premenopausal women has been a question of concern. HE4 is not usually above normal values in patients with endometriosis [11]. In our cohort, the majority of false positive results of CA125 were found in patients with endometriomas (96 patients), while only one patient with elevated HE4 harbored ovarian endometriosis. Patients with ovarian endometriosis accounted for $14 \%$ and $19 \%$ of false positive results of ROMA and $\mathrm{CPH}-\mathrm{I}$, which is to be expected considering that both probabilistic indexes include the value of CA125. A work on differential diagnosis of EOC and ovarian endometriosis in premenopausal woman suggests that HE4 has higher accuracy and AUC than CA125 and ROMA [34].

Most patients with false negative results of probabilistic indexes and tumor markers presented initial stages of ovarian cancer, whose preoperative diagnosis still remains challenging. In our cohort, probabilistic indexes performed similarly, and ROMA performed better than tumor markers alone for diagnosis of Stage I EOC. Moreover, in the analysis of the subgroup of patients with an inconclusive diagnosis by ultrasound, the ROMA model showed the highest AUC. This might suggest probabilistic models (especially ROMA) are particularly useful in cases whose diagnosis by ultrasound is challenging. Kaijser et al. [35] conclude that HE4 and ROMA, as secondary tests, do not seem useful for classification of adnexal tumors after subjective assessment with transvaginal ultrasonography by experienced Level III examiners. Further studies in these specific subpopulations are needed to confirm these data.

\subsection{The Role of Isolated CA125 for Preoperative Assessment od Adnexal Tumors}

HE4 performed better in terms of AUC, sensitivity and specificity than CA125. As mentioned before, in premenopausal women, HE4 was even equivalent to ROMA and CPH-I in ROC analysis. This raises the question of whether we can do without CA125 for preoperative assessment of patients with suspected ovarian cancer. Jacob et al. concluded that HE4 might be used alone, without the benefit of adding ROMA to the preoperative setting [36] and Montagnana et al. [32] concluded that the dual marker combination of HE4 and CA125 does not show better performance than HE4 alone in premenopausal women. However, our data show that nearly $10 \%$ of patients with EOC or MCO presented an isolated positivity of CA125 (with negative HE4). Moreover, except for premenopausal women (HE4 was equivalent to CPH-I and ROMA), and Stage I EOC (HE4 was equivalent to $\mathrm{CPH}-\mathrm{I}), \mathrm{CPH}-\mathrm{I}$ and ROMA performed better than HE4 alone. Therefore, even if CA125 shows less specificity and sensitivity than HE4, it adds valuable information for the diagnosis of EOC or MCO. 


\subsection{Beyond CA125 and HE4: The Role of Novel Tumor Markers and Ultrasound}

Some studies have evaluated the role of other biomarkers for diagnosis of ovarian cancer, with variable results. The OVA1 index is an FDA-approved multivariate index assay that has been considered a good triage tool for patients with ovarian masses requiring surgery, although its low specificity and its worse diagnostic performance in premenopausal women have raised some concerns [37-41]. A second generation multivariate index assay $\left(\right.$ Overa ${ }^{\circledR}$ ) showed an improvement in terms of specificity $(61.9 \%$ ) with similar sensitivity $(91.3 \%)$ to Ova1 [41]. Han et al. suggested in 2018 that a four biomarker panel including CA125, HE4, E-CAD, and IL-6 might be a potential tool for diagnosis of serous ovarian cancer at early stages [42]. Zhu et al. recently proposed a new algorithm including HE4, CA125 and thymidine kinase 1; the authors suggest that this algorithm has better diagnostic performance than ROMA, and report higher AUCs than those identified in our cohort, for both pre- and post-menopausal women [43]. In exchange, Moore et al. report that the addition of other biomarkers to HE4 and CA125 does not improve diagnostic performance [38]. Further validation studies are needed to confirm these results.

Ultrasound adds valuable information to preoperative assessment of adnexal masses, thus several models based on ultrasound features have been described, such as RMI, IOTA-LR1 and LR2 or ADNEX [44]. Notably, IOTA-LR1 and LR2 models have shown good diagnostic performance, with reported AUCs of 0.96 and 0.95 respectively, higher than those obtained for tumor-marker based models in our cohort [45]. ADNEX model combines ultrasound features with CA125, and has a sensitivity of $96.5 \%$, a specificity of $71.3 \%$, and a 0.94 AUC [46]. The ESGO/ISUOG/IOTA/ESGE Consensus Statement on Preoperative Diagnosis of Ovarian Tumors reports that ultrasound-based diagnostic models (IOTA simple rules or ADNEX) are preferable to CA125 level, HE4 level or ROMA [28]. In 2015, an algorithm including HE4, menopausal status and ultrasound findings was described, with higher sensitivity but less specificity than ROMA algorithm [47]. To the best of our knowledge, to date, no algorithms including ultrasonographic items and HE4 have been validated. Further studies are needed to determine whether a probabilistic model combining clinical information, ultrasound features and both HE4 and CA125 might offer the best diagnostic approach.

\subsection{Strengths and Limitations of the Present Study}

We believe that the most relevant limitation of the present study is the retrospective nature of the analysis. Patients were recruited in a single institution, which might be a limitation in terms of the external validity of the study. Nevertheless, we included a high number of patients from the same hospital, which allowed us to achieve a high degree of uniformity in the procedures performed (blood sample extraction and processing, pathologic analysis, ultrasound performance). To the best of our knowledge, this is the first single institution study that includes more than 1000 patients comparing the role of CPH-I and ROMA indexes for preoperative assessing of adnexal tumors. We expect that data arising from this study will help to improve diagnostic protocols for preoperative evaluation of adnexal masses. However, further prospective studies are needed to determine the best diagnostic algorithm.

\section{Conclusions}

Improving preoperative diagnosis of patients with ovarian malignancies is essential to enhancing their survival. CA125 and HE4, and the probabilistic indexes ROMA and CPH-I have been proposed as a tool for preoperative evaluation of patients with adnexal tumors. According to our data, ROMA and CPH-I perform significantly better than tumor markers alone to identify patients harboring ovarian malignancies in overall population. In premenopausal women, HE4 is equivalent to probabilistic indexes. ROMA performs better for the diagnosis of Stage I EOC and for patients harboring adnexal masses whose diagnosis by ultrasound remains inconclusive. 
ROMA and CPH-I can be helpful to assess the risk of malignancy of adnexal masses, especially when expert ultrasound examination is not available or when the diagnosis is challenging (early-stage ovarian cancer, inconclusive ultrasonographic evaluation). An algorithm including CA125, HE4 and ultrasound features might improve the diagnostic approach. Further prospective studies are needed to determine the best diagnostic algorithm for patients with ovarian malignancies.

Supplementary Materials: The following are available online at https:/ /www.mdpi.com/article/10 .3390/diagnostics12010226/s1, Table S1: ROC analysis of Ca125, He4, ROMA and CPH for diagnosis of EOC or MCO. $p$-values of the comparison between ROC curves. Table S2: false negative results for tumor markers and probabilistic indexes.

Author Contributions: Conceptualization, P.F.; methodology, P.F., A.G., N.C.-D., M.M.; formal analysis, G.C., A.G., N.C.-D. and M.A.A.; investigation, N.C.-D., A.G., M.M., B.D.-F., N.A., B.S., A.S., L.G., M.A.A., A.T., E.F.-G. and J.P. validation, P.F. and A.T.; writing-original draft preparation, N.C.-D. and A.G.; writing-review and editing, P.F., A.T., M.A.A.; supervision, P.F., A.T.; contributed equally and share first co-authorship, A.G. and N.C.-D.; share senior co-authorship, P.F. and A.T. All authors have read and agreed to the published version of the manuscript.

Funding: This research received no external funding.

Institutional Review Board Statement: The study was conducted according to the guidelines of the Declaration of Helsinki, and approved by the Ethics Committee of Hospital Clínic de Barcelona (HCB_2015_0571, 14 July 2015).

Informed Consent Statement: Informed consent was obtained from all subjects involved in the study.

Data Availability Statement: The data presented in this study are available on request from the corresponding author.

Acknowledgments: In memoriam of Rafael Molina.

Conflicts of Interest: The authors declare no conflict of interest.

\section{References}

1. Bray, F.; Ferlay, J.; Soerjomataram, I.; Siegel, R.L.; Torre, L.A.; Jemal, A. Global cancer statistics 2018: GLOBOCAN estimates of incidence and mortality worldwide for 36 cancers in 185 countries. CA. Cancer J. Clin. 2018, 68, 394-424. [CrossRef]

2. Timmerman, D.; Van Calster, B.; Testa, A.; Savelli, L.; Fischerova, D.; Froyman, W.; Wynants, L.; Van Holsbeke, C.; Epstein, E.; Franchi, D.; et al. Predicting the risk of malignancy in adnexal masses based on the Simple Rules from the International Ovarian Tumor Analysis group. Am. J. Obstet. Gynecol. 2016, 214, 424-437. [CrossRef] [PubMed]

3. Giede, K.C.; Kieser, K.; Dodge, J.; Rosen, B. Who should operate on patients with ovarian cancer? An evidence-based review. Gynecol. Oncol. 2005, 99, 447-461. [CrossRef]

4. Piovano, E.; Cavallero, C.; Fuso, L.; Viora, E.; Ferrero, A.; Gregori, G.; Grillo, C.; Macchi, C.; Mengozzi, G.; Mitidieri, M.; et al. Diagnostic accuracy and cost-effectiveness of different strategies to triage women with adnexal masses: A prospective study. Ultrasound Obstet. Gynecol. 2017, 50, 395-403. [CrossRef]

5. Sayasneh, A.; Kaijser, J.; Preisler, J.; Smith, A.A.; Raslan, F.; Johnson, S.; Husicka, R.; Ferrara, L.; Stalder, C.; Ghaem-Maghami, S.; et al. Accuracy of ultrasonography performed by examiners with varied training and experience in predicting specific pathology of adnexal masses. Ultrasound Obstet. Gynecol. 2015, 45, 605-612. [CrossRef]

6. Alcázar, J.L.; Pascual, M.A.; Graupera, B.; Aubá, M.; Errasti, T.; Olartecoechea, B.; Ruiz-Zambrana, A.; Hereter, L.; Ajossa, S.; Guerriero, S. External validation of IOTA simple descriptors and simple rules for classifying adnexal masses. Ultrasound Obstet. Gynecol. 2016, 48, 397-402. [CrossRef] [PubMed]

7. Knafel, A.; Banas, T.; Nocun, A.; Wiechec, M.; Jach, R.; Ludwin, A.; Kabzinska-Turek, M.; Pietrus, M.; Pitynski, K. The prospective external validation of International Ovarian Tumor Analysis (IOTA) simple rules in the hands of level i and II examiners. Ultraschall der Medizin 2016, 37, 516-523. [CrossRef]

8. Nunes, N.; Ambler, G.; Foo, X.; Naftalin, J.; Widschwendter, M.; Jurkovic, D. Use of IOTA simple rules for diagnosis of ovarian cancer: Meta-analysis. Ultrasound Obstet. Gynecol. 2014, 44, 503-514. [CrossRef]

9. Maggino, T.; Gadducci, A.; D’Addario, V.; Pecorelli, S.; Lissoni, A.; Stella, M.; Romagnolo, C.; Federghini, M.; Zucca, S.; Trio, D.; et al. Prospective multicenter study on CA 125 in postmenopausal pelvic masses. Gynecol. Oncol. 1994, 54, 117-123. [CrossRef] [PubMed]

10. Jacobs, I.; Robert, C.B., Jr. The CA125 tumour-associate antigen: A review of the literature. Hum. Reprod. 1989, 4, 1-12. [CrossRef] 
11. Moore, R.G.; Brown, A.K.; Miller, M.C.; Skates, S.; Allard, W.J.; Verch, T.; Steinhoff, M.; Messerlian, G.; DiSilvestro, P.; Granai, C.O.; et al. The use of multiple novel tumor biomarkers for the detection of ovarian carcinoma in patients with a pelvic mass. Gynecol. Oncol. 2008, 108, 402-408. [CrossRef]

12. Galgano, M.T.; Hampton, G.M.; Frierson, H.F. Comprehensive analysis of HE4 expression in normal and malignant human tissues. Mod. Pathol. 2006, 19, 847-853. [CrossRef]

13. Moore, R.G.; Miller, M.C.; Eklund, E.E.; Lu, K.H.; Bast, R.C.; Lambert-Messerlian, G. Serum levels of the ovarian cancer biomarker HE4 are decreased in pregnancy and increase with age. Am. J. Obstet. Gynecol. 2012, 206, 349-e1. [CrossRef] [PubMed]

14. Escudero, J.M.; Auge, J.M.; Filella, X.; Torne, A.; Pahisa, J.; Molina, R. Comparison of serum human epididymis protein 4 with cancer antigen 125 as a tumor marker in patients with malignant and nonmalignant diseases. Clin. Chem. 2011, 57, 1534-1544. [CrossRef]

15. Moore, R.G.; McMeekin, D.S.; Brown, A.K.; DiSilvestro, P.; Miller, M.C.; Allard, W.J.; Gajewski, W.; Kurman, R.; Bast, R.C.; Skates, S.J. A novel multiple marker bioassay utilizing HE4 and CA125 for the prediction of ovarian cancer in patients with a pelvic mass. Gynecol. Oncol. 2009, 112, 40-46. [CrossRef] [PubMed]

16. Dayyani, F.; Uhlig, S.; Colson, B.; Simon, K.; Rolny, V.; Morgenstern, D.; Schlumbrecht, M. Diagnostic Performance of Risk of Ovarian Malignancy Algorithm Against CA125 and HE4 in Connection with Ovarian Cancer: A Meta-analysis. Int. J. Gynecol. Cancer 2016, 26, 1586-1593. [CrossRef] [PubMed]

17. Karlsen, M.A.; Høgdall, E.V.S.; Christensen, I.J.; Borgfeldt, C.; Kalapotharakos, G.; Zdrazilova-Dubska, L.; Chovanec, J.; Lok, C.A.R.; Stiekema, A.; Mutz-Dehbalaie, I.; et al. A novel diagnostic index combining HE4, CA125 and age may improve triage of women with suspected ovarian cancer-An international multicenter study in women with an ovarian mass. Gynecol. Oncol. 2015, 138, 640-646. [CrossRef] [PubMed]

18. Minar, L.; Felsinger, M.; Cermakova, Z.; Zlamal, F.; Bienertova-Vasku, J. Comparison of the Copenhagen Index versus ROMA for the preoperative assessment of women with ovarian tumors. Int. J. Gynecol. Obstet. 2018, 140, 241-246. [CrossRef]

19. Han, K.H.; Park, N.H.; Kim, J.J.; Kim, S.; Kim, H.S.; Lee, M.; Song, Y.S. The power of the risk of ovarian malignancy algorithm considering menopausal status: A comparison with CA 125 and HE4. J. Gynecol. Oncol. 2019, 30, e83. [CrossRef]

20. Zhen, S.; Bian, L.; Chang, L.; Gao, X. Comparison of serum human epididymis protein 4 and carbohydrate antigen 125 as markers in ovarian cancer: A meta-analysis. Mol. Clin. Oncol. 2014, 2, 559-566. [CrossRef]

21. Van Gorp, T.; Cadron, I.; Despierre, E.; Daemen, A.; Leunen, K.; Amant, F.; Timmerman, D.; De Moor, B.; Vergote, I. HE4 and CA125 as a diagnostic test in ovarian cancer: Prospective validation of the Risk of Ovarian Malignancy Algorithm. Br. J. Cancer 2011, 104, 863-870. [CrossRef]

22. Yoshida, A.; Derchain, S.F.; Pitta, D.R.; De Angelo Andrade, L.A.L.; Sarian, L.O. Comparing the Copenhagen Index (CPH-I) and Risk of Ovarian Malignancy Algorithm (ROMA): Two equivalent ways to differentiate malignant from benign ovarian tumors before surgery? Gynecol. Oncol. 2016, 140, 481-485. [CrossRef]

23. Tran, D.T.; Vo, V.K.; Le, M.T.; Chuang, L.; Nguyen, V.Q.H. Copenhagen Index versus ROMA in preoperative ovarian malignancy risk stratification: Result from the first Vietnamese prospective cohort study. Gynecol. Oncol. 2021, 162, 113-119. [CrossRef] [PubMed]

24. Fishman, D.A.; Cohen, L.; Blank, S.V.; Shulman, L.; Singh, D.; Bozorgi, K.; Tamura, R.; Timor-Tritsch, I.; Schwartz, P.E. The role of ultrasound evaluation in the detection of early-stage epithelial ovarian cancer. Am. J. Obstet. Gynecol. 2005, 192, $1214-1221$. [CrossRef]

25. Kurman, R.J.; Carcangiu, M.L.; Harrington, C.S.; Young, R.H. WHO Classification of Tumours of Female Reproductive Organs, 4th ed.; IARC Publications: Lyon, France, 2014; p. 307.

26. DeLong, E.R.; DeLong, D.M.; Clarke-Pearson, D.L. Comparing the Areas under Two or More Correlated Receiver Operating Characteristic Curves: A Nonparametric Approach. Biometrics 1988, 44, 837-845. [CrossRef] [PubMed]

27. Timmerman, D.; Testa, A.C.; Bourne, T.; Ameye, L.; Jurkovic, D.; Van Holsbeke, C.; Paladini, D.; Van Calster, B.; Vergote, I.; Van Huffel, S.; et al. Simple ultrasound-based rules for the diagnosis of ovarian cancer. Ultrasound Obstet. Gynecol. 2008, 31, 681-690. [CrossRef]

28. Timmerman, D.; Planchamp, F.; Bourne, T.; Landolfo, C.; du Bois, A.; Chiva, L.; Cibula, D.; Concin, N.; Fischerova, D.; Froyman, W.; et al. ESGO/ISUOG/IOTA/ESGE Consensus Statement on preoperative diagnosis of ovarian tumors. Ultrasound Obstet. Gynecol. 2021, 58, 148-168. [CrossRef]

29. Moore, R.G.; Jabre-Raughley, M.; Brown, A.K.; Robison, K.M.; Miller, M.C.; Allard, W.J.; Kurman, R.J.; Bast, R.C.; Skates, S.J. Comparison of a novel multiple marker assay vs the Risk of Malignancy Index for the prediction of epithelial ovarian cancer in patients with a pelvic mass. Am. J. Obstet. Gynecol. 2010, 203, 228. [CrossRef]

30. Melo, Â.; Veríssimo, R.; Farinha, M.; Martins, N.N.; Martins, F.N. Discriminative value of CA-125, HE4, Risk of Malignancy Index II (RMI-II) and Risk of Malignancy Algorithm (ROMA) in the differential diagnosis of pelvic masses: Conclusions from a referral Centre in Portugal. J. Obstet. Gynaecol. 2018, 38, 1140-1145. [CrossRef]

31. Sandri, M.T.; Bottari, F.; Franchi, D.; Boveri, S.; Candiani, M.; Ronzoni, S.; Peiretti, M.; Radice, D.; Passerini, R.; Sideri, M. Comparison of HE4, CA125 and ROMA algorithm in women with a pelvic mass: Correlation with pathological outcome. Gynecol. Oncol. 2013, 128, 233-238. [CrossRef] [PubMed] 
32. Montagnana, M.; Danese, E.; Ruzzenente, O.; Bresciani, V.; Nuzzo, T.; Gelati, M.; Salvagno, G.L.; Franchi, M.; Lippi, G.; Guidi, G.C. The ROMA (Risk of Ovarian Malignancy Algorithm) for estimating the risk of epithelial ovarian cancer in women presenting with pelvic mass: Is it really useful? Clin. Chem. Lab. Med. 2011, 49, 521-525. [CrossRef] [PubMed]

33. Suri, A.; Perumal, V.; Ammalli, P.; Suryan, V.; Bansal, S.K. Diagnostic measures comparison for ovarian malignancy risk in Epithelial ovarian cancer patients: A meta-analysis. Sci. Rep. 2021, 11, 17308. [CrossRef] [PubMed]

34. Nikolova, T.; Zivadinovic, R.; Evtimovska, N.; Klisarovska, V.; Stanojevic, M.; Georgievska, J.; Nikolova, N. Diagnostic performance of human epididymis protein 4 compared to a combination of biophysical and biochemical markers to differentiate ovarian endometriosis from epithelial ovarian cancer in premenopausal women. J. Obstet. Gynaecol. Res. 2017, 43, 1870-1879. [CrossRef]

35. Kaijser, J.; Van Gorp, T.; Smet, M.E.; Van Holsbeke, C.; Sayasneh, A.; Epstein, E.; Bourne, T.; Vergote, I.; Van Calster, B.; Timmerman, D. Are serum HE4 or ROMA scores useful to experienced examiners for improving characterization of adnexal masses after transvaginal ultrasonography? Ultrasound Obstet. Gynecol. 2014, 43, 89-97. [CrossRef]

36. Jacob, F.; Meier, M.; Caduff, R.; Goldstein, D.; Pochechueva, T.; Hacker, N.; Fink, D.; Heinzelmann-Schwarz, V. No benefit from combining HE4 and CA125 as ovarian tumor markers in a clinical setting. Gynecol. Oncol. 2011, 121, 487-491. [CrossRef] [PubMed]

37. Terzic, M.; Aimagambetova, G.; Norton, M.; Della Corte, L.; Marín-Buck, A.; Lisón, J.F.; Amer-Cuenca, J.J.; Zito, G.; Garzon, S.; Caruso, S.; et al. Scoring systems for the evaluation of adnexal masses nature: Current knowledge and clinical applications. $J$. Obstet. Gynaecol. 2021, 41, 340-347. [CrossRef]

38. Moore, R.G.; Blackman, A.; Miller, M.C.; Robison, K.; DiSilvestro, P.A.; Eklund, E.E.; Strongin, R.; Messerlian, G. Multiple biomarker algorithms to predict epithelial ovarian cancer in women with a pelvic mass: Can additional makers improve performance? Gynecol. Oncol. 2019, 154, 150-155. [CrossRef]

39. Ueland, F. A Perspective on Ovarian Cancer Biomarkers: Past, Present and Yet-To-Come. Diagnostics 2017, 7, 14. [CrossRef] [PubMed]

40. Dunton, C.J.; Hutchcraft, M.L.; Bullock, R.G.; Northrop, L.E.; Ueland, F.R. Salvaging detection of early-stage ovarian malignancies when ca125 is not informative. Diagnostics 2021, 11, 1440. [CrossRef]

41. Coleman, R.L.; Herzog, T.J.; Chan, D.W.; Munroe, D.G.; Pappas, T.C.; Smith, A.; Zhang, Z.; Wolf, J. Validation of a secondgeneration multivariate index assay for malignancy risk of adnexal masses. Am. J. Obstet. Gynecol. 2016, 215, 82-e1. [CrossRef]

42. Han, C.; Bellone, S.; Siegel, E.R.; Altwerger, G.; Menderes, G.; Bonazzoli, E.; Egawa-Takata, T.; Pettinella, F.; Bianchi, A.; Riccio, F.; et al. A novel multiple biomarker panel for the early detection of high-grade serous ovarian carcinoma. Gynecol. Oncol. 2018, 149, 585-591. [CrossRef]

43. Zhu, C.; Zhang, N.; Zhong, A.; Xiao, K.; Lu, R.; Guo, L. A combined strategy of TK1, HE4 and CA125 shows better diagnostic performance than risk of ovarian malignancy algorithm (ROMA) in ovarian carcinoma. Clin. Chim. Acta 2022, 524, 43-50. [CrossRef] [PubMed]

44. Moro, F.; Esposito, R.; Landolfo, C.; Froyman, W.; Timmerman, D.; Bourne, T.; Scambia, G.; Valentin, L.; Testa, A.C. Ultrasound evaluation of ovarian masses and assessment of the extension of ovarian malignancy. Br. J. Radiol. 2021, 94, 20201375. [CrossRef] [PubMed]

45. Kaijser, J.; Bourne, T.; Valentin, L.; Sayasneh, A.; Van Holsbeke, C.; Vergote, I.; Testa, A.C.; Franchi, D.; Van Calster, B.; Timmerman, D. Improving strategies for diagnosing ovarian cancer: A summary of the International Ovarian Tumor Analysis (IOTA) studies. Ultrasound Obstet. Gynecol. 2013, 41, 9-20. [CrossRef] [PubMed]

46. Sayasneh, A.; Ferrara, L.; De Cock, B.; Saso, S.; Al-Memar, M.; Johnson, S.; Kaijser, J.; Carvalho, J.; Husicka, R.; Smith, A.; et al Evaluating the risk of ovarian cancer before surgery using the ADNEX model: A multicentre external validation study. Br. J. Cancer 2016, 115, 542-548. [CrossRef]

47. Wilailak, S.; Chan, K.K.L.; Chen, C.A.; Nam, J.H.; Ochiai, K.; Aw, T.C.; Sabaratnam, S.; Hebbar, S.; Sickan, J.; Schodin, B.A.; et al. Distinguishing benign from malignant pelvic mass utilizing an algorithm with HE4, menopausal status, and ultrasound findings. J. Gynecol. Oncol. 2015, 26, 46-53. [CrossRef] 